\title{
Supporting Digital Transformation in Small and Medium-sized Enterprises: A Procedure Model Involving Publicly Funded Support Units
}

\author{
Benjamin Barann* \\ barann@ercis.de
}

\author{
Andreas Hermann* \\ hermann@ercis.de
}

Ann-Kristin Cordes* cordes@ercis.de

* University of Muenster - ERCIS

\author{
Friedrich Chasin* \\ chasin@ercis.de
}

Jörg Becker*

becker@ercis.de

\begin{abstract}
Digital Transformation (DT) considers the continuous digitalization process of a company, which uses digital and data-driven innovation to improve existing processes, change distinct business model (BM) elements, or reinvent its BM entirely. Large companies position themselves at the frontline of the DT, while small and medium-sized enterprises (SMEs) are challenged by resource constraints and missing guidance on realizing benefits of DT. This situation threatens SMEs as big players increasingly utilize DT to enter markets that have been traditionally reserved to SMEs. Extant research on the ways SMEs can effectively participate in DT is limited. Against this background, this article builds upon prior research and a focus group discussion to propose a procedure model, which enables $D T$ in SME by involving publicly funded support units. These units help SMEs in understanding and structuring the potentials of digital and data-driven innovation.
\end{abstract}

\section{Introduction}

Technology has always been changing the way companies operate. Nowadays, companies started to look for easy ways to implement digitalization projects so that they can keep up with their competitors and satisfy their customers [50]. Between 2012 and 2013, the term digital transformation (DT), which explains this continuous process (cf. chapter 3.1), gained popularity in media (e.g. Google Trends) and academic research [e.g. 26]. In DT, companies are not only focusing on the exploitation of technology to improve operational efficiency but also on the exploration of further innovation potentials [5]. Exploring the potentials of digital innovations to change BM elements or to implement a completely new BM has become quite common in the age of digitalization [42]. Such digital innovations go along with the generation of vast and diverse amounts of data [23, 37, 51]. Nowadays, companies go beyond the mere storing of data. They understand the potential value and explicitly consider data-driven innovation $[5,18,20,21,23]$.
While SMEs are more flexible, faster or less constrained $[10,31]$, resources constraints and knowledge gaps often hinder these companies to evaluate and implement digitalization opportunities [5, $10,18,25,31]$. This raises the question: How can SMEs utilize their limited resources to drive digital and datadriven innovation effectively and limit the risk of failure that can result from enforcing the DT of their business?

To get started with this complex undertaking, companies often demand the support of external consultants [50]. However, SMEs often cannot afford costly third-party support [18]. Instead, SMEs can receive support from publicly funded support units. Such a support unit is an organizational entity typically comprising several academic and non-academic institutions funded by the government [e.g. 40]. While being free of charge, these help SMEs to increase their awareness of digital innovation potentials in workshops, teach the fundamental skills in training and support the implementation of selected innovations in projects [e.g. $33,55]$.

In addition to this support, the idea of systematically tackling the DT by provisioning some sort of procedure model has become a common way in the respective research domain (see chapter 3.3). This paper argues, that while larger companies may be able to experiment with different innovation projects, smaller companies require a more practical approach focusing on feasible and tangible goals. SMEs require an approach that wraps the underlying complexity of DT projects into manageable and easily understood action items, rather than abstract frameworks. Such an approach exploits the advantageous characteristics of SMEs in terms of being more flexible, faster or less constrained [10, 31]. However, there is only limited research presenting procedures that are specifically suited to SMEs. What is more, the support of publicly funded support units is not explicitly regarded by any procedure (see chapter 3.3).

With this in mind, the objective is to develop a procedure model that (1) incorporates an intensive interface to publicly funded support units and (2) tackles DT in pragmatic steps adhering to the peculiarities posed by the nature of SMEs. 
The remainder of this paper is structured as follows: In chapter two, the research approach is presented. Chapter three derives requirements for the procedure model and chapter four describes the procedure model. Finally, the article is concluded by a short discussion, the evaluation and an outlook on further research.

\section{Research Approach}

In order to elaborate the research background of the subject area, existing literature on DT and business model innovation (BMI) was reviewed first following a semi-structured process by Lindgren and Jansson [36].

Second, in March 2018, an independent two-hour focus group discussion with experts of the "MittelstandDigital" initiative (www.mittelstanddigital.de), which comprises various "Mittelstand 4.0" competence centers and agencies, was conducted to collect data for the refinement and evaluation cycle [53]. The discussion took place during an event organized collaboratively by the "Agentur Kommunikation" (www.kommunikation-mittelstand.digital) and the "Agentur Handel" (www.handel-mittelstand.digital). Members of all Mittelstand 4.0 support units (competence centers), which interact with SMEs on a regular basis to support their DT efforts, were invited. Furthermore, members of all four support agencies, which guide the support units, participated. In total, 30 experts participated in the discussion: One person from a controlling unit of the initiative, 15 participants from four supportive agencies, and 13 members from eight different competence centers. To ensure the exchange of thoughts between the competence centers and agencies, the participants were divided into eight mixed groups and had to address the question: "What is your secret hint for the successful implementation of digitalization projects?" The results were presented to facilitate discussions between the groups. For this article, the results were further coded to derive initial requirements for the procedure model.

Third, a structured literature review following Webster and Watson [56] and vom Brocke et al. [9] was conducted to investigate how publicly funded support units can support SMEs in their DT. The review used two search term categories each internally connected with an OR-operator and considered the titles, abstracts, and keywords (if applicable): (1) ((business model innovation, digital transformation, digital innovation, data-driven innovation), (publicly funded, support unit, competence center, competency center, competence centre, competency centre)) AND (2) SME, small and medium. Furthermore, the German translations were added to the search string. The 379 resulting articles from the databases EBSCOHost (99), ScienceDirect (204), AISNet (13), and Scopus (63) were then filtered for duplicates. The remaining 325 results were then analyzed by reviewing their titles and abstracts. Based on this analysis, publications, which did not focus on the subject area, were excluded. Next, the remaining publications were read in detail. 15 relevant publications with full-text access mentioning procedures or process steps for SME were identified. Using the insights from these articles, the requirements were further refined and enriched (cf. chapter 3.3).

Fourth, the resulting model was synthesized (cf. chapter 5) according to the definition of research synthesis by Cooper and Hedges [13]. Therefore, the requirements derived from the focus group discussion were used to provide an initial structure for the model. Next, the existing literature was screened to derive further requirements. Finally, the existing procedures were analyzed and iteratively integrated into the model by merging, adapting, and adding new steps.

Finally, the resulting model was evaluated against the derived requirements and a design-accompanied evaluation was conducted [4] (see. chapter 6). The goal of this evaluation was to ensure that the model could trigger an ongoing DT in SMEs.

\section{Research Background}

\subsection{Digital Transformation and SMEs}

DT can be regarded as an iterative organizational process, which comprises incremental and disruptive changes enabled by digital technology. It comprises the ability to adapt a BM with or to new technologies and technological innovations in the socio-technical environment, which have an impact on the operations and the customer experience [20, 26, 28, 35, 39, 43].

Enterprise transformation can happen incrementally when expected, or in a "big bang" [18]. Besides the utilization of digital potential and the creation of a digital value network and a digital customer experience, the DT offers opportunities to change or add BM elements [43]. It might also have an impact on the entire $\mathrm{BM}$. Indeed, DT describes a process that moves beyond catching up with the status quo. It "is a technologyinduced change on many levels in the organization that includes both the exploitation of digital technologies to improve existing processes, and the exploration of digital innovation, which can potentially transform the business model" [5]. This corresponds to the BM design themes of efficiency and novelty and the topic of organizational ambidexterity [38]. Furthermore, DT is concerned with the digitalization of the whole organization [1]. This allows the generation and exchange of large quantities of data [23, 37, 51]. Therefore, DT is also fueled by data-driven innovations $[5,18,20,21,23]$ 
In a survey with 417 organizations in Switzerland and Germany, prior research has shown that most companies possess a medium-level DT maturity and, thus, are in the process of digitally transforming their enterprise [5]. While bigger companies are starting to understand the opportunities and challenges of the DT, SMEs are still struggling and face challenges on their path to a DT [19, 23, 31]. SMEs employ fewer than 250 employees and do not exceed a 50 million EUR annual turnover [16]. Most of SMEs cannot yet be considered as BM innovators [3]. The reasons for this are within others missing resources such as personnel capacity, skills, and funding [10, 18, 25, 31]. SMEs are therefore reliant on the success of DT projects [18]. However, it is hard for SMEs to identify beneficial DT projects [23]. For SME entrepreneurs to be successful in DT, it is not enough to integrate current technologies [35]. Arbitrary technological innovation may not guarantee firm success, because technology in itself has no intrinsic value [61]. What is more, the information and qualification requirements of SMEs differ from those of larger companies [40]. In addition, SMEs have special requirements concerning the supporting tools [18]. This is aggravated by the fact that dedicated, simple tools that are tailored to SME requirements are barely available [25]. Finally, SMEs cannot consider all innovation activities necessary for the successful realization of an innovation on their own [10]. However, paid external support, e.g. from market research institutes [31], is often too expensive for SMEs [18]. Still, SMEs are less formalized, less constrained, faster and more flexible $[10,31]$. Thus, they generally have the capacity to even implement radical innovation [10], especially if they are able to capitalize on the guidance provided by publicly funded support units.

\subsection{Digital and Data-driven Innovation}

While innovation in a BM may arise due to different reasons [38], this article focusses on technologyenabled changes in the context of the DT. These changes can be classified into the overlapping terms digital and data-driven innovation.

Digital innovation is a process by which a digital business design takes advantage of technology to implement "new strategic options" [48, 54]. A digital innovation can be incremental or radical and does not necessarily result in a change within the BM [18]. In fact, there is no common agreement in regard to which level of innovation is required to be considered as BMI [57]. Therefore, digital innovation is discussed on three levels [cf. 1, 6]: (1) The first possibility to digitally transform a business is to digitalize the operating model and optimize processes [6, 12, 15, 54]. This is also an important enabler, as digital and automated processes are required for new BMs [23]. (2) Second, a company can digitally rethink their BM [6] by using digital technology to adapt, improve or extend a component of the existing BM $[8,23,38]$. One prominent example is the value proposition, which has a direct impact on the customer value and profits $[23,54]$. (3) The third and most disruptive form of digital innovation is the development of a completely new digital BM $[15,54]$ or the utilization of secondary value creation opportunities, which are not part of the main business. The latter option is called BM sophistication [31]. For example, a company may introduce new individualized (digital) products or services or combinations of both [8, $12,15,23]$. Furthermore, a company can identify new revenue streams beyond the main business or decrease its existing costs by motivating stakeholders to take over the costs [31]. For example, a company could monetize novel digital assets on top of their existing BM [8].

Data-driven innovation is a specialized form of digital innovation that can also be seen on the three levels. (1) A data-driven innovation takes advantage of data and analytics to reach the growth and well-being of an organization [59]. In addition, data is said to be a "key resource in digitization", which can "enable or support business activities" [23]. (2) Next, data can also be used to adapt, improve or extend a component of the existing BM. The utilization of data as a key resource is said to result in a data-driven BM [45]. However, there was no clear definition of this term in the past $[22,45]$. While some scholars already consider data as a key resource on the first level of data-driven innovation [23], such a BM will just progressively become more datadriven [45]. Therefore, this article argues that a datadriven BM comprises at least one distinctive BM element, which is enabled by data as a key resource and/or data analytics as a key activity. For example, a company might enable innovative service BMs on top of the existing value propositions [59]. (3) Finally, data as a key resource and/or analytics as key activity can enable entirely new BMs [1]. Indeed, big data businesses can not only be considered as a data user but also as a data supplier or data facilitator [11]. Examples for this are BMs that provide data, analytics, software/platforms, or expertise as a service $[11,45]$.

In sum, this article defines data-driven innovation as the use of data and analytics for improved data-driven processes, refined/extend data-driven BM elements, or completely new data-driven BMs [11, 22, 30, 45, 51]. Data-driven BMs are defined as a subset of digital BMs, which consider data as a key resource or analytics as a key activity to refine, extend, or rethink the enterprise. 


\subsection{Procedure Models for Digital Transformation in SMEs}

SMEs are often not aware of innovation potentials [31], struggle to understand what to digitalize, which technology to use, how to prioritize goals, and which organizational changes (e.g. skills and roles) are required [23]. This highlights the need for systematic guidance through publicly funded support units.

Through the first semi-structured literature review, the subject area could be understood and an overview of the current state of research could be gained. Existing literature is extensive and the topic has been discussed for years. Seven relevant and representative contributions in this field without an explicit focus on SME but a rather general applicability could be identified. These gave a good overview of relevant process steps $[7,14,27,28,41,55,57,60]$. The idea of systematically tackling the DT with procedure models has become a common way in the respective research domain (see below). Research and practice have yielded to a number of conceptual models that aim at structuring the DT into a set of abstract steps.

Through the literature review on SME specific procedure models, 15 relevant contributions could be identified [10, 18, 19, 23, 25, 31, 33, 35, 38, 42, 46, 47, $49,55,58]$. The identified articles differ in various ways and focus on different aspects. First, contributions tackle DT on a strategic, tactical or operational level. Some of the models are rather characterized by a focus on strategic aspects $[7,14,18,19,41,60]$. For example, Van Goolen et al. [19] emphasize the importance of congruency between digital innovations and company goals and the focus is put on high-level strategic goals and company visions. Others have a mixed focus including both strategic and tactical/operational elements [25, 27]. While all three levels exist in the literature, there is a tendency towards a strategic level.

Second, a majority of the identified contributions reflect a similar underlying logic. As the term "transformation" inherently suggests, most of the contributions cover the following abstract phases in one way or another: Analysis of a current as-is state, definition of the desired to-be state, an alignment/synthesize of the as-is and to-be states and an actual implementation and monitoring phase $[23,25,27$, 41, 42, 46, 49, 57, 58, 60].

Finally, only three publications present a procedure model for SME as a central outcome. However, they focus on BMI $[19,58]$ or a specific industry [18] only. Most of the other publications only mention generic process steps as part of their line of argumentation. For example, some of the BMI publications deal with the tools, which can be used by SME to support the generic innovation phases [23, 42, 46, 49]. Others discuss the actual approach of case study companies [24, 25], investigate the behavior of SMEs in this context [35, $38]$, consider the advantages of open innovation [10, 47], or discuss specific opportunities for BMI [31] without focusing on concrete process steps. In particular, no procedure does explicitly incorporate the support of external support units. If any, the collaboration with external entities is sometimes mentioned [18, 19, 23, 42]. Only two publications of support units shortly mention their abstract steps to aid digitalization efforts in specific domains $[33,55]$.

The following consequences can be drawn from the literature review. First, existing procedure models that approach the DT, regardless of whether the focus is on SMEs or not, are mainly conceptual in nature. In other words, ideal-theoretic steps are hardly aligned to actual practical needs of SMEs. Even though the underlying logic of those contributions is reasonable for DT in general as well as for SME, they neglect idiosyncratic peculiarities that are important for DT projects of SMEs in practice [49]. Second, a process model incorporating external support units does not exist. Hence, in the following, a set of special requirements for such a procedure is presented.

\section{Requirements for a Procedure Model to Support Digital Transformation in SMEs}

To define an initial set of eight requirements (R1R8), the data collected from the focus group discussion was coded independently from literature. In addition, the extant research identified through the literature review supports and complements those requirements. In sum, the following 11 requirements for the procedure model could be defined:

R1 - Integration of External Supporters: Incorporate external supporters such as competence centers or research institutions, which can help SMEs to understand and implement DT initiatives. Similar to this, the literature talks about collaboration with SME helpers, innovation labs, research institutions, and other intermediaries [18, 19, 23, 42].

R2 - Provision of Practical Orientation: Provide domain-specific orientation for DT by guiding the SMEs through current trends and demonstrate their importance based on practical examples [cf. 19, 41, 58]. Literature suggests providing light, simple, easy-to-use, agile, and practical tools [25, 42, 49]. Therefore, a procedure should go beyond the conceptual level and help to develop overviews incorporating domainspecific knowledge, best-practice, real-life examples, and pre-filled tools [25, 42].

R3 - Creation of a Supportive Environment: Create an awareness of the basic conditions, which need to be ensured before an implementation project starts. 
R4 - Consideration of Tangible Goals: Support SMEs with the requirements and needs analysis, which enables them to clearly define measurable goals.

R5 - Provision of an Individual Roadmap: Support SMEs with the creation of a simple roadmap, considering their current state of DT and a mix of quickwins as well as sustainable innovations [cf. 23].

R6 - Enabling a Stepwise Implementation: Provide a cyclic step-by-step approach, which helps SMEs to implement feasible objectives one after another. This allows the sequential implementation to encounter the lack of resources [25, 42]. Still, the procedure should allow different entry points [42] and provide the possibility to flexibly adjust the goals [10].

R7 - Identification of Opportunities: Support SMEs to identify different possible solutions for a specific goal and select the most appropriate one. Thus, different business cases should be compared [42].

R8 - Assisting Reflection \& Measurement: Supporting SMEs in understanding and evaluating (un)successful digitization efforts in order to generate best practice and motivate further projects.

R9 - Balancing Strategy and Operation: Lead to actions, yet, ensure a strategic fit [25, 42].

R10 - Supporting all Levels of Digital and Datadriven Innovation: Balance simple stepwise and radical innovations [10]. Thus, it should support all levels of digital and data-driven innovation [23, 25].

R11 - Consideration of Open Innovation: Consider the building of communities, in which SMEs can help each other [42]. To drive the performance of their innovation process [10,19], ideas and resources should be shared between internal and external entities [29].

\section{A Procedure Model to Enable Digital Transformation in SMEs}

Within existing literature, the innovation process is typically described in several steps covering e.g. the idea generation, idea evaluation, and idea selection, experimentation, refinement, commercial launch, and monitoring and controlling [e.g. 14, 57, 60]. Some procedures focus more on the orientation and consider the actual implementation of an innovation almost as a black-box [7, 19, 41]. These models cover steps that help to position a company within the digitalization by understanding the current $\mathrm{BM}$ and identifying digitalization potentials. In contrast, other models support both the orientation and the (iterative) implementation of innovation [18, 27, 33, 55, 58]. Covering both phases is important as digitalization requires a business- and an IT-perspective [23].

Therefore, the designed procedure considers both phases (see Fig. 1). In other words, the procedure comprises two separate innovation cycles [e.g. 18], which focus on different questions: An "Orientation" cycle (Phase I) and an "Iterative Transformation" cycle (Phase II). The first phase comprises two detail processes and defines a roadmap of business goals for DT. These goals are based on the current situation of the SME, ideas for digital or data-driven innovations and the strategic goals of the SME. In the second phase, an iterative transformation process is triggered, which comprises three detail processes. One iteration might implement one or several progressively enabled goals by considering complementary opportunities. Also, a goal might be placed back to a new position on the roadmap, if its implementation is ahead of time or beyond the available capacity [14]. Despite the resource constraints, SMEs have a "relatively low barrier to innovate" and require a practical approach, which is agile and not too strategic [25, 49]. To enable this flexibility and to ensure a sustainable competitive advantage [19], the second phase can be terminated with a back-loop to the first. This allows the roadmap resulting from Phase I to be skimmed regularly $[18,19]$. In sum, the two phases represent a "recursive, virtuous cycle" [38], which not only enables the efficiencycentered exploitation of technology but also the exploration of BMI in terms of novelty-centered [38] and sophistication-centered innovation [31]. Thus, they consider all levels of digital and data-driven innovation.

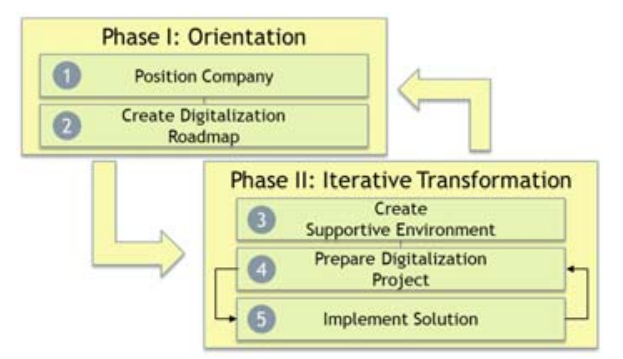

Fig. 1: A Procedure Model for DT in SME

Throughout the procedure, SMEs are closely supported by a support unit. Its involvement is depicted with an asterisk next to the names of the process steps. A second asterisk highlights the possibility of support in a participative format with several SMEs.

\subsection{Position Company}

In the first step, a company has to be positioned within the digitalization [41] (see Fig. 2.1). Therefore, a planning team [27] comprising of the SME's CEO [18] and members of the support unit needs to be established.

Next, the planning team has to understand the current $B M[7,18,23,31,43,49,52]$. Furthermore, the as-is situation needs to be analyzed to identify 
challenges, risks, or changed customer expectations beyond the industry level $[27,34,41,43,46,49,52,57$, $58,60]$. Here, the support unit can offer benchmarks and training to support the management's reflection [35]. Also, it should synthesize the results of this step [60].

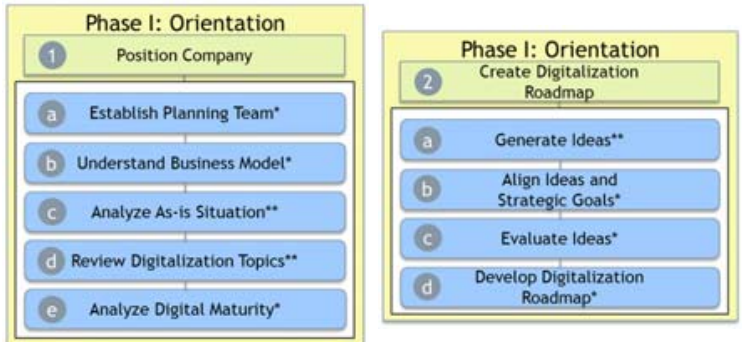

Fig. 2: Detail Processes: (1) Position Company and (2) Create Digitalization Roadmap

According to the focus group, SMEs need some orientation and a general understanding in the beginning. To support SMEs in this regard, the current digitalization topics need to be reviewed [7, 19, 23, 27, $28,42,43,58]$ by the external support unit. Here the support unit acts as a complement for market researchers and strategic units employed by larger companies [31]. Based on this review, an aggregated overview of digitalization topics presenting the domainspecific digitalization trends, value drivers, scenarios, impacts, and benchmarks should be created [7, 25, 41, 49]. This review should follow the open innovation principle, which builds and involves a network of formal and informal partnerships [10, 35, 47]. The support unit should then offer talks focusing on the identified topics to improve the understanding of SMEs [23].

Finally, the support unit should analyze the SME's digital maturity [23, 28, 33]. This could be done with existing maturity models [23].

\subsection{Create Digitalization Roadmap}

Next, a well-informed digital roadmap has to be created (see Fig 2.2). Therefore, ideas for the different levels of digital or data-driven innovation should be generated in a workshop organized by the support unit [14, 19, 27, 28, 43, 46, 57, 58]. It should involve several SMEs $[10,47]$ and initially present aggregated results from the first detail process to discuss motivating scenarios [41]. Next, the workshop should follow two approaches for idea generation. First, considering a bottom-up approach (i.e. starting at the existing business processes, new BMs and technologies, the available data, and potential analytics), it should identify "low hanging fruits" [23]. Also, potentials to collect and store data should be gathered, even if the actual innovation potential is not yet clear [23]. Second, a top-down approach (i.e. starting from the existing BM and the underlying strategy) might uncover further disruptive changes [23] and BM sophistication opportunities [31]. Both approaches are important drivers of the digitalization [23] and lead to ideas spanning the different levels of digital and data-driven innovation.

Second, in close collaboration with the CEO [57], the support unit should align the ideas with the strategic goals [19, 42]. This is important as strategic goals have an impact on the innovation outcome in SME, even if an explicitly formulated strategy does not exist [24]. Also, open innovation requires this step to be sustainable [2].

Third, the aligned ideas need to be evaluated, clustered, and refined by the support unit to determine the potential impact areas, benefits, problems, and their feasibility, considering the current situation $[14,19,41$, $43,46,52,57,58]$. As there may be several ways to technically implement an idea, they should be first evaluated on a business level, postponing a detailed feasibility-analysis [41] to a later point (see chapter 5.4). The result of this step is a list of business goals connected to the generated ideas [19].

Finally, a digitalization roadmap ordering and prioritizing the refined goals is developed collaboratively $[10,18,23,41,43,58]$. Simple goals should be prioritized that introduce enabling technologies for more complex goals (cf. chapter 5.5). While disruptive BM changes might be too complex to get started [23], they should be considered, as they could threaten the existing business [23]. The support unit can develop guidelines, which help to choose the goals with the highest success rates [19]. As the procedure aims to be flexible and not too strategic, the roadmap only serves as DT vision [23] and not as a clearly defined strategy. Therefore, the roadmap only contains the business goals instead of clear objectives and responsibilities [10] related to a gap- and feasibilityanalysis [41].

\subsection{Create Supportive Environment}

Next, the iterative transformation [18] can be triggered (see Fig. 3.3). As a first step, a supportive environment needs to be created. This was stated to be an important success factor by the focus group. The employees and the management need to be aware of the digitalization to support initial initiatives [5]. Therefore, the support unit needs to ensure that the SMEs understand the different goals and their added value by providing practical examples.

Furthermore, the focus group, scholars [28, 32, 44], and practitioners [52] stated that it is important to involve employees and integrate the necessary people in the DT process. Therefore, a team such as an open innovation steering committee could be formed, which 
also strengthens the open innovation and business capabilities $[10,35]$. The latter enable the SMEs to "seize opportunities to develop and grow business" [35].

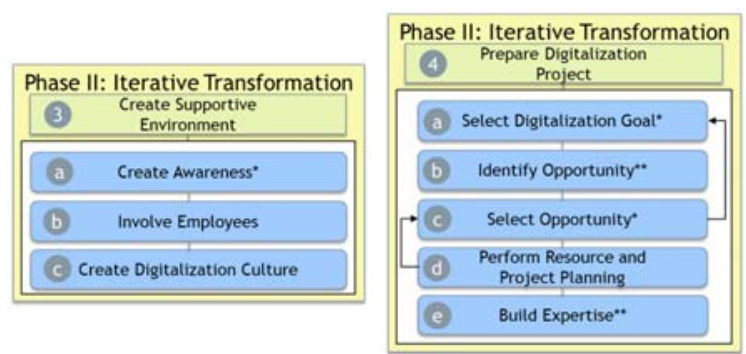

Fig. 3: Detail Processes: (3) Create Supportive Culture and (4) Prepare Digitalization Project

Finally, an enabling DT culture needs to be created [5, 17, 19, 21, 23, 41]. Especially radical BMI requires a supportive culture [38]. In sum, the focus group named trust, openness, an error-culture, communication, internal and external networking, and appreciation. The SMEs need a connective capacity and should be able to absorb external ideas (i.e. open innovation) [10].

\subsection{Prepare Digitalization Project}

Afterward, the planning team and the involved employees need to prepare the digitalization project. As a first step, the digitalization goal with the highest priority is selected from the roadmap [18] (see Fig. 3.4).

For the selected goal, different opportunities need to be identified by the support unit [e.g. 14, 19, 27, 42]. In contrast to the process step "Generate Ideas" (2.a), a set of exchangeable solutions for a selected goal is generated, which propose concrete action items [23]. To support this, participative formats in the sense of open innovation can be pursued [10,47] if several SMEs share common goals. Also, creativity [46] or BM modeling techniques [58] can be used. The different solutions can be outlined as a list of issues related to the business goal the required and available technologies or data sources, and the concrete implementation idea. Also, simple intermediate solutions, use cases, or user stories can be produced [23].

The third process step "select opportunity" takes the identified opportunity space, evaluates it in detail and iteratively refines the most promising ones [14, 19, 41, 57]. This task is guided by the support unit and requires detailed input from the SME. Within others, it needs to be evaluated if an opportunity is a suitable starting point [19] and appropriate for the issue at hand [23]. Also, a "cost-benefit analysis, an impact analysis of existing practices, offerings and resources, a risk analysis, and an analysis of constraints" needs to be performed [41]. Thereby, opportunities with high risk, the probability of failure or high costs need to be identified, refined or discarded [14]. E.g. the implementation might be too expensive because technical prerequisites are not fulfilled [52]. Also, the SME should perform an environmental analysis considering the competitive structures [57] and the "organization's ability to change should be considered" [41]. If no feasible solution can be identified, it might be necessary to select a different goal from the roadmap, first [19].

In case a suitable opportunity exists, detailed resource and project planning are performed. Subteams responsible for the next steps should be formed. Based on the developed use cases [23], a competence gap analysis should be performed [58]. Different scenarios including the internal realization through targeted up-skilling or recruiting and the support through implementation partners need to be considered $[18,32,52]$. After this, a final decision can be made concerning the funding and the scheduling of the project $[19,52]$. If the opportunity is found to be infeasible in this step, another opportunity needs to be reconsidered.

Finally, the required expertise to develop and sustain the digital or data-driven innovation is built by training efforts offered by the support unit.

\subsection{Implement Solution}

Finally, the digital or data-driven innovation gets iteratively implemented [18, e.g. 19, 57] (see Fig. 4). The process first experimentally designs the solution in a draft or prototype to increase its concreteness and test its executability $[14,19,24,27,42,57,58,60]$. This step may use existing platform solutions [18] or even designs previously developed by the support unit.

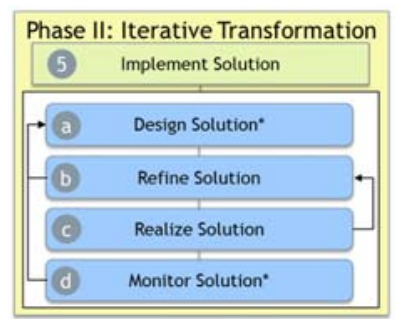

Fig. 4: Detail Process: Implement Solution

Next, the solution is iteratively refined. Within others, it is verified, validated, aligned with the BM, and external market value is created (e.g. by involving possible stakeholders) $[14,18,27,42,57,60]$. The open innovation principle can also support this process step [10]. It might be necessary to go back to the design of the solution to solve challenges that were uncovered.

In the third process step, the refined solution gets realized (i.e. rolled-out) [e.g. 27, 42, 43, 57, 58]. Therefore, the required strategic and organizational 
changes have to be made [60]. This includes adjustments to the resources, capabilities, structures, maintenance mechanisms, core processes, and all other elements that might be impacted by the innovation [14, 60]. Furthermore, "buy-in and acceptance" have to be generated [14] in a pilot test [58, 60]. It might be necessary to tweak the innovation further by going back to the refinement of the solution $[14,18]$.

Finally, the realized solution should be monitored by the support unit. This step evaluates if the solution fulfills the desired goal [23, 28, 43, 57, 58]. If necessary, the process step implements the required corrective actions [57] and ensures that the SME can grow through innovation [42]. Furthermore, the impacts need to be understood [27] and the results, as well as the lessons learned, should be documented [27, 58]. The gathered insights might enable back-loops at the end of the detail process [58]. First, a newly introduced digital or datadriven innovation could serve as an enabler for the direct implementation of another goal. New ideas might come up, which then result in the next innovation cycle or require a reevaluation of the roadmap [14, 23]. Next, secondary value-capturing opportunities (cf. chapter 3.2) might be enabled by the innovation [31]. Third, in the case of a BMI, there might be further changes to the $\mathrm{BM}$, which are required due to the impact of the innovation [34, 38]. Finally, a better understanding of the BMI process might result in the capability to proactively innovate the $\mathrm{BM}$ in prospective projects [34]. Through the new capabilities and experiences, further strategic changes might be pursued [35]. Indeed, it has been shown that SMEs innovate BM elements sequentially [25]. Also, capability building should continue to allow the SME to adapt to changing conditions [35]. Through the improved capabilities and experiences, further DT efforts could happen with a reduced involvement of the support unit.

\section{Evaluation and Conclusion}

In the modern digital economy, SMEs are being constantly challenged by the novel and often disruptive digital enterprises that continue to change the ways of doing business. This motivates to bring the DT of SMEs into focus. However, through a structured literature review, this article revealed that only three prior publications present a procedure model for DT in SMEs as a central outcome of their research $[18,19,58]$. What is more, publicly funded support units are not explicitly incorporated in these models. Besides, most of the other publications just focus on an abstract excerpt of the steps. Thus, to contribute to the knowledge gap, this article developed an approach that wraps the underlying complexity of DT projects into manageable and easily understood action items guided by publicly funded support units. This was done by deriving requirements in a focus group discussion and by synthesizing a procedural model from literature. The derived requirements and the designed model adhere to the unique aspects of DT projects in SMEs. The assistance of the support units is adapted to SMEs as it starts with the basics, builds standardized practical instruments for SMEs of a specific industry, incorporates standardized participative formats for a better scaling, and considers a neutral position for selecting a solution. While the outcomes of DT will certainly differ between small and medium enterprises, the procedure remains the same.

Tab. 1: Requirements Matching

\begin{tabular}{|l|l|l|}
\hline Requirement & Explicit & Implicit \\
\hline $\begin{array}{l}\text { Integration of External } \\
\text { Supporters }\end{array}$ & $\begin{array}{l}\text { 1.a, 1.b, 1.c, 1.d, 1.e, 2.a, 2.b, } \\
\text { 2.c, 2.d, 3.a, 4.b, 4.c, 4.e, 5.d }\end{array}$ & $4 . \mathrm{a}, 5 . \mathrm{a}$ \\
\hline $\begin{array}{l}\text { Provision of Practical } \\
\text { Orientation }\end{array}$ & 1.d, 1.e, 2.a, 3.a, 4.b, 5.a & $1 . \mathrm{c}$ \\
\hline $\begin{array}{l}\text { Creation of a Supportive } \\
\text { Environment }\end{array}$ & $3 . \mathrm{a}, 3 . \mathrm{b}, 3 . \mathrm{c}$ & $1 . \mathrm{a}, 4 . \mathrm{d}, 4 . \mathrm{e}$ \\
\hline $\begin{array}{l}\text { Consideration of Tangible } \\
\text { Goals }\end{array}$ & 2.c, 4.a, 4.b & $\begin{array}{l}\text { 1.b, 1.c, 1.d, } \\
1 . \mathrm{e}, 2 . \mathrm{a}, 2 . \mathrm{b}\end{array}$ \\
\hline $\begin{array}{l}\text { Provision of an Individual } \\
\text { Roadmap }\end{array}$ & 2.a, 2.b, 2.c, 2.d & $1 . \mathrm{e}, 5 . \mathrm{d}$ \\
\hline $\begin{array}{l}\text { Enabling a Stepwise } \\
\text { Implementation }\end{array}$ & 2.d, 4.a, 5.d & By design \\
\hline $\begin{array}{l}\text { Identification of } \\
\text { Opportunities }\end{array}$ & 4.b, 4.c & 4.d \\
\hline $\begin{array}{l}\text { Assisting Reflection \& } \\
\text { Measurement }\end{array}$ & $3 . \mathrm{c}, 5 . \mathrm{d}$ & 5.a, 5.b, 5.c \\
\hline $\begin{array}{l}\text { Balancing Strategy and } \\
\text { Operation }\end{array}$ & $2 . \mathrm{b}, 5 . \mathrm{c}$ & By design \\
\hline $\begin{array}{l}\text { Supporting all Levels of } \\
\text { Digital and Data-driven } \\
\text { Innovation }\end{array}$ & $2 . \mathrm{a}, 5 . \mathrm{d}$ & By design \\
\hline $\begin{array}{l}\text { Consideration of Open } \\
\text { Innovation }\end{array}$ & $1 . \mathrm{d}, 2 . \mathrm{a}, 3 . \mathrm{c}, 4 . \mathrm{b}, 5 . \mathrm{b}$ & $1 . \mathrm{c}, 5 . \mathrm{a}$ \\
\hline
\end{tabular}

The evaluation of the designed procedure is twofold. First, the procedure was matched against the derived requirements (see Tab. 1). The model accounts for requirements R1-R11 either in an explicit or implicit way. In an explicit case, at least one process step address a particular requirement. In an implicit case, the models underlying logic implies the fulfillment of the requirement. For instance, the procedure accounts for a reasonable balance between strategy and operation by structuring the DT into two dedicated phases.

Second, the conceptual design followed an iterative procedure with alternating development and discussion phases in the context of a "Mittelstand 4.0" competence center (i.e. design accompanied-evaluation [4]). In the course of informative and participative events including meetings, presentations, and expert dialogs, the procedure was discussed and refined. However, one limitation of this research is that the procedure has not yet been empirically tested. Now, the application of the procedure would allow further evaluation cycles.

Further research should develop a method to identify domain-specific DT topics (1.d) organized into maturity levels to allow a practical evaluation of the digitalization 
maturity (1.e). Next, domain-specific mappings between topics and opportunities (4.b) should be developed (e.g., a framework, which matches goals with concrete data-driven innovations). Also, solutions for these potentials can be gathered and offered as an encapsulated service on a collaborative web-platform (5). Finally, evaluation methods (5.d) and KPIs, for both, the topics and opportunities might provide sufficient empirical evidence for the effectiveness of the proposed instruments for DT in SMEs to encourage SMEs to arm themselves with these for the future.

\section{Acknowledgments}

This work was created in the context of a project that received funding from the BMWi under the grant agreement 01MF17011D.

\section{References}

[1] Appelfeller, W., and C. Feldmann, Die digitale Transformation des Unternehmens - Systematischer Leitfaden mit zehn Elementen zur Strukturierung und Reifegradmessung, Gabler Verlag, 2018.

[2] Banu, G.S., A. Dumitrescu, A.A. Purcărea, and S.W. Isărescu, "Defining Open Innovation Concept Using Business Process Modeling”, Procedia Technology 22, 2016, pp. 1020-1027.

[3] Barjak, F., A. Niedermann, and P. Perrett, "The Need for Innovations in Business Models - Final Policy Brief”, 2014. https://ec.europa.eu/research/innovation-union/pdf/expertgroups/ERIAB-BMI PB new business models.pdf [4] Becker, J., P. Delfmann, R. Knackstedt, and D. Kuropka, "Konfigurative Referenzmodellierung", In J. Becker and R.

Knackstedt, eds., Wissensmanagement mit Referenzmodellen. Physica-Verlag HD, Heidelberg, 2002, 25-144.

[5] Berghaus, S., and A. Back, "Stages in Digital Business Transformation: Results of an Empirical Maturity Study", MCIS 2016 Proceedings, (2016).

[6] Berman, S.J., "Digital transformation: opportunities to create new business models", Strategy \& Leadership 40(2), 2012, pp. 16-24.

[7] Bleicher, J., and H. Stanley, "Digitization as a Catalyst for Business Model Innovation a Three-step Approach to Facilitating Economic Success", Journal of Business Management(12), 2016, pp. 62-71.

[8] Bonnet, D., and G. Westermann, "The Best Digital Business Models Put Evolution Before Revolution”, Harvard Business Review(20), 2015.

[9] vom Brocke, J., M. Fay, M. Böhm, and V. Haltenhof, "Creating a Market Analytics Tool that Marketers LOVE to Use: A Case of Digital Transformation at Beiersdorf", In G. Oswald and M. Kleinemeier, eds., Shaping the Digital Enterprise: Trends and Use Cases in Digital Innovation and Transformation. Springer International Publishing, Cham, 2017, 197-217.

[10] Brunswicker, S., and F. Ehrenmann, "Managing open innovation in SMEs: A good practice example of a german software firm", International Journal of Industrial Engineering and Management 4(1), 2013, pp. 33-41. [11] Bulger, M., G. Taylor, and R. Schroeder, "Data-Driven
Business Models: Challenges and Opportunities of Big Data", Oxford Internet Institute(September), 2014, pp. 1-74. [12] Büllingen, F., "Digitale Geschäftsmodelle: Wieso sie wichtig sind und was sie ausmacht", Digitale Geschäftsmodelle: Themenheft Mittelstand Digital, 2017. https://www.bmwi.de/Redaktion/DE/Publikationen/Mittelsta $\mathrm{nd} /$ mittelstand-digital-digitale-geschaeftsmodelle.pdf [13] Cooper, H., L. V Hedges, and J.C. Valentine, The handbook of research synthesis and meta-analysis, Russell Sage Foundation, 2009.

[14] Desouza, K.C., C. Dombrowski, Y. Awazu, et al., "Crafting organizational innovation processes", Innovation 11(1), 2009, pp. 6-33.

[15] Dirzus, D., and C. Gülpen, "Mehr Wertschöpfung durch neue Geschäftsmodelle", Wissenschaft trifft Praxis, 2017. https://www.mittelstand-

digital.de/MD/Redaktion/DE/Publikationen/Wissenschafttrifft-Praxis/magazin-wissenschaft-trifft-praxis-ausgabe8 [16] European Union, "Commission Recommendation of 6 May 2003 concerning the definition of micro, small and medium-sized enterprises", Official Journal of the European Union, 2003, pp. 36-41.

[17] Fitzgerald, M., N. Kruschwitz, D. Bonnet, and M. Welch, "Embracing digital technology: A new strategic imperative", MIT sloan management review 55(2), 2014. [18] Goerzig, D., and T. Bauernhansl, "Enterprise Architectures for the Digital Transformation in Small and Medium-sized Enterprises", Procedia CIRP 67, 2018, pp. 540-545.

[19] Van Goolen, R., H. Evers, and C. Lammens, "International Innovation Labs: An Innovation Meeting Ground between SMEs and Business Schools", Procedia Economics and Finance 12, 2014, pp. 184-190.

[20] Gray, J., and B. Rumpe, "Models for the digital transformation", Software \& Systems Modeling 16(2), 2017, pp. 307-308.

[21] Haffke, I., B. Kalgovas, and A. Benlian, "The Role of the $\mathrm{CIO}$ and the CDO in an Organization's Digital

Transformation", ICIS 2016 Proceedings, (2016), 1-20. [22] Hartmann, P., M. Zaki, ... N.F.-A.T. of D.-D., and U. 2014, Big data for big business? A taxonomy of data-driven business models used by start-up firms, 2014.

[23] Heberle, A., W. Löwe, A. Gustafsson, and V. Vorrei, "Digitalization Canvas - Towards identifying digitalization use cases and projects", Journal of Universal Computer Science 23(11), 2017, pp. 1070-1097.

[24] Heikkilä, M., H. Bouwman, and J. Heikkilä, "From strategic goals to business model innovation paths: an exploratory study", Journal of Small Business and Enterprise Development 25(1), 2018, pp. 107-128.

[25] Heikkilä, M., H. Bouwman, J. Heikkilä, T. Haaker, C.L. Nicolas, and A. Riedl, "Business Model Innovation Paths and Tools", 29th Bled eConference Digital Economy, (2016).

[26] Henriette, E., M. Feki, and I. Boughzala, "The Shape of Digital Transformation: A Systematic Literature Review", Mediterranean Conference on Information Systems (MCIS) Proceedings(October), 2015, pp. 1-13.

[27] Hunke, F., S. Seebacher, R. Schuritz, and A. Illi, "Towards a Process Model for Data-Driven Business Model Innovation”, 2017 IEEE 19th Conference on Business Informatics (CBI), IEEE (2017), 150-157. 
[28] Ismail, M.H., M. Khater, and M. Zaki, Digital Business Transformation and Strategy: What Do We Know So Far?, 2017.

[29] Johannsson, M., A. Wen, B. Kraetzig, et al., "Space and Open Innovation: Potential, limitations and conditions of success", Acta Astronautica 115, 2015, pp. 173-184.

[30] Kastner, R., "Datengetriebene Geschäftsmodelle - Was steckt dahinter?", Huffington Post, 2017.

[31] Kesting, P., and F. Günzel-Jensen, "SMEs and new ventures need business model sophistication", Business Horizons 58(3), 2015, pp. 285-293.

[32] Kohnke, O., "It's Not Just About Technology: The People Side of Digitization", In G. Oswald and M.

Kleinemeier, eds., Shaping the Digital Enterprise - Trends and Use Cases in Digital Innovation and Transformation. Springer International Publishing, 2017, 69-91.

[33] Kraut, A., J. Hicking, S. Ackermann, and Y. Krolle, "Ermittlung des Digitalisierungsgrads von KMU in NRW", ZWF Zeitschrift für wirtschaftlichen Fabrikbetrieb 113(1-2), 2018, pp. 92-95.

[34] Laudien, S.M., and B. Daxböck, "Business model innovation processes of average market players: a qualitative-empirical analysis", $R \& D$ Management 47(3), 2017, pp. 420-430.

[35] Li, L., F. Su, W. Zhang, and J.-Y. Mao, "Digital transformation by SME entrepreneurs: A capability perspective”, Information Systems Journal, 2017.

[36] Lindgren, I., and G. Jansson, "Electronic services in the public sector: A conceptual framework", Government Information Quarterly 30(2), 2013, pp. 163-172.

[37] Loebbecke, C., and A. Picot, "Reflections on societal and business model transformation arising from digitization and big data analytics: A research agenda", The Journal of Strategic Information Systems 24(3), 2015, pp. 149-157.

[38] Loon, M., and R. Chik, "Efficiency-centered, innovation-enabling business models of high tech SMEs: Evidence from Hong Kong", Asia Pacific Journal of Management, 2018.

[39] Matt, C., T. Hess, and A. Benlian, "Digital Transformation Strategies", Business and Information Systems Engineering 57(5), 2015, pp. 339-343.

[40] Müller, E., and H. Hopf, "Competence Center for the Digital Transformation in Small and Medium-Sized Enterprises", Procedia Manufacturing 11, 2017, pp. 1495 1500.

[41] Parviainen, P., M. Tihinen, J. Kääriäinen, and S. Teppola, "Tackling the digitalization challenge: how to benefit from digitalization in practice", International Journal of Information Systems and Project Management 5(1), 2017, pp. 63-77.

[42] Reuver, M. De, A. Athanasopoulou, T. Haaker, M. Roelfsema, and A. Riedl, "Designing an ICT tooling platform to support SME business model innovation: Results of a first design cycle", 29th Bled eConference Proceedings, (2016), 1-15.

[43] Schallmo, D., and A. Rusnjak, "Roadmap zur Digitalen Transformation von Geschäftsmodellen", In Digitale Transformation von Geschäftsmodellen. Springer Fachmedien Wiesbaden, Wiesbaden, 2017, 1-31.

[44] Schuchmann, D., and S. Seufert, "Corporate Learning in Times of Digital Transformation: A Conceptual Framework and Service Portfolio for the Learning Function in Banking Organisations", International Journal of Advanced Corporate Learning (iJAC) 8(1), 2015, pp. 31. [45] Schuritz, R., and G. Satzger, "Patterns of Data-Infused Business Model Innovation", 2016 IEEE 18th Conference on Business Informatics (CBI), IEEE (2016), 133-142.

[46] Seidenstricker, S., E. Rauch, and P. Dallasega, "Industrie-4.0-Geschäftsmodellinnovation für KMU", ZWF Zeitschrift für wirtschaftlichen Fabrikbetrieb 112(9), 2017, pp. 616-620.

[47] Silviana, B.G., "Open innovation model: enabling the market uptake of innovation", Procedia Manufacturing 22, 2018, pp. 893-899.

[48] Slywotzky, A.J., D. Morrison, and K. Weber, How digital is your business?, Crown Business, 2001.

[49] Smit, S., G. Fragidis, S. Handschuh, and A. Koumpis, "Business Model Boutique: A Prêt-à-Porter Solution for Business Model Innovation in SMEs", In 2016, 579-587. [50] Soares, P.A. de S., and E. Müller, "Die Rattenfänger von Digitalien”, manager magazin, 2018, 68-77.

[51] Sorescu, A., "Data-Driven Business Model Innovation", Journal of Product Innovation Management 34(5), 2017, pp. 691-696.

[52] Thiessen, T., J. Oltersdorf, K. Kühn, and C. Augusti, “In 5 Schritten zur Digitalisierung”, 2017.

https://kommunikation-

mittelstand.digital/content/uploads/2017/06/Leitfaden-

Digitalisierung-in-5-Schritten.pdf

[53] Tremblay, M.C., A.R. Hevner, and D.J. Berndt, "The Use of Focus Groups in Design Science Research", In 2010, $121-143$

[54] Wall, B., H. Jagdev, and J. Browne, "A review of eBusiness and digital business - applications, models and trends", Production Planning \& Control 18(3), 2007, pp. 239-260.

[55] Wank, A., S. Adolph, O. Anokhin, A. Arndt, R. Anderl, and J. Metternich, "Using a Learning Factory Approach to Transfer Industrie 4.0 Approaches to Small- and Mediumsized Enterprises", Procedia CIRP 54, 2016, pp. 89-94. [56] Webster, J., and R.T. Watson, "Analyzing the Past to Prepare for the Future: Writing a Literature Review", MIS Quarterly 26(2), 2002, pp. xiii-xxiii.

[57] Wirtz, B.W., and M.-J. Thomas, "Design und Entwicklung der Business Model-Innovation", In Kompendium Geschäftsmodell-Innovation. Springer Fachmedien Wiesbaden, Wiesbaden, 2014, 31-49.

[58] Wißotzki, M., and K. Sandkuhl, "The Digital Business Architect - Towards Method Support for Digital Innovation and Transformation", In 2017, 352-362.

[59] Zolnowski, A., T. Christiansen, and J. Gudat, "Business model transformation patterns of data-driven innovations", 24th European Conference on Information Systems, ECIS 2016(June), 2016.

[60] Zott, C., and R. Amit, "Business Model Innovation: Towards a Process Perspective", In C. Shalley, M.A. Hitt and J. Zhou, eds., The Oxford Handbook of Creativity, Innovation, and Entrepreneurship. Oxford University Press, New York, NY, 2015.

[61] Zott, C., R. Amit, and L. Massa, "The Business Model: Recent Developments and Future Research", Journal of Management 37(4), 2011, pp. 1019-1042. 